\title{
Nível de atividade física e apoio social dos pais e amigos em escolares da rede pública
}

\author{
Level of physical activity and social support of parents and friends in schools of the \\ public network
}

\section{AUTORES \\ Wesley Marcos Daniel Todisco ${ }^{1}$ (D) \\ Flávio Ricardo Guilherme ${ }^{2}$ (iD) \\ Carlos Alexandre Molena-Fernandes ${ }^{1}$ (iD \\ Stevan Ricardo dos Santo ${ }^{1}$ (D) \\ Rafael José Fernandes Ravagnani ${ }^{1}$ (ID \\ Wlady Alves Barbosa ${ }^{1}$ (D) \\ Fabrício Boscolo Del Vecchio ${ }^{3}$ (D) \\ Wilson Rinaldi ${ }^{2}$ ID \\ 1 Universidade Estadual do Paraná - Campus Paranavaí - Departamento de Educação Física. Paranavaí, Paraná, Brasil. \\ 2 Universidade Estadual de Maringá - Programa de Pós-Graduação em Educacão Física Associado UEM/UEL. Maringá, Paraná, Brasil. \\ 3 Universidade Federal de Pelotas - Escola Superior de Educação Física. Pelotas, RS, Brasil.}

\section{CONTATO}

Stevan Ricardo dos Santos

stevan_ricardo@hotmail.com

Rua Francisco Isidoro Oliveira 367. Paranavaí, PR, Brasil. CEP:87709-090.

DOI

10.12820/rbafs.v.22n5p457-63

\section{$(1) \Theta \Theta$}

Copyright: This is an open-access article distributed under the terms of the Creative Commons Attribution License ${ }^{\circledR}$, which permits unrestricted use, distribution, and reproduction in any medium, provided that the original author and source are credited.

\begin{abstract}
RESUMO
O estudo teve como objetivo analisar a associação entre apoio social e nível de atividade física em escolares. Pesquisa com delineamento transversal, caracterizou-se como censo escolar, visto que envolveu toda população escolar do Ensino Fundamental II e Médio, de 10 a 18 anos de idade, de oito escolas públicas existentes na cidade de Paranavaí, Paraná ( $\mathrm{n}=2.759,14,3 \pm 3,3$ anos, 50,5\% meninas e 49,5\% meninos. O nível de atividade física foi analisado por uma adaptação do questionário Self Administered Physical Activity Checklist, e o ponto de corte para inadequação do nível de atividade física foi <300 minutos por semana. O apoio social de pais e amigos para a prática de atividade física foi avaliado por meio da escala de Apoio Social para Atividade Física em Adolescentes (ASAFA), sendo considerado como inadequado quando das respostas foram nunca e raramente. As análises estatísticas foram feitas por meio do Statistical Package for a Social Science (SPSS), versão 20.0, considerando-se um nível de significância de $5 \%$. Os resultados mostraram que escolares com apoio social inadequado dos pais, dos amigos ou de ambos, possuíam, respectivamente, 2,0 (IC95\%: 1,6-2,6), 1,5 (IC95\%: 1,2-2,9) e 1,6 (IC95\%: 1,2-2,1) mais de chances de serem insuficientes ativos em relação aos que tinham o apoio social adequado. Existe associação entre nível de atividade física e apoio social de pais e amigos em escolares da rede pública de ensino. Nesse sentido, sugere-se às escolas que sejam desenvolvidas iniciativas para conscientização de pais e amigos quanto a sua importância com vistas à adoção da prática de atividade física em adolescentes.
\end{abstract}

Palavras-chave: Inatividade física; Apoio social; Adolescentes.

\section{ABSTRACT}

The study aimed to verify the existence of an association between social support and level of physical activity in schoolchildren. A cross-sectional survey was characterized as a school census, since it involved all the school population of primary and secondary education from 10 to 18 years old from eight public schools in the city of Paranavai, Paraná( $n=2.759,14,3 \pm 3.3$ years, $50.5 \%$ girls and $49.5 \%$ boys). The level of physical activity was analyzed by an adaptation of the Self Administered Physical Activity Checklist, and the cut-off point for inadequate level of physical activity was $<300 \mathrm{~min}$ per week. The social support of parents and friends for the practice of physical activity was evaluated through the Social Support for Physical Activity in Adolescents (ASAFA) scale, being considered as inadequate when the answers were never and rarely. Statistical analyzes were performed using the Statistical Package for Social Science (SPSS), version 20.0, considering $p<0.05$. The results show that students with inadequate social support from parents, friends or both had, 2.0 (95\%CI: 1.6-2.6), 1.5 (95\%CI: 1.2-2.9) and 1.6 (95\%CI: 1.2-2.1) more chances to be insufficient active compared to those who have adequate support. There is an association between level of physical activity and social support of parents and friends in schoolchildren. In this sense, it is suggested that schools develop initiatives to raise awareness of parents and friends regarding their importance in order to adopt the practice of physical activity in adolescents.

Keywords: Physical inactivity; Social support; Adolescents.

\section{Introdução}

Atividade física é qualquer movimento corporal produzido pelos músculos esqueléticos que resulta em gasto energético maiores que o nível de repouso ${ }^{1}$ : Para crianças e adolescentes serem fisicamente ativos eles devem realizar no mínimo 300 minutos por semana de atividades físicas de intensidades moderada a vigorosa ${ }^{2}$.
A prática de atividade física na adolescência está relacionada com benefícios na saúde esquelética (conteúdo mineral e densidade óssea), controle da pressão sanguínea, dislipidemia obesidade e síndrome metabólica, além de melhoria nas habilidades motoras, no desenvolvimento psicológico e no nível de relação social ${ }^{3-8}$. Apesar do conhecimento científico sobre os benefícios 
da atividade física na adolescência para a saúde, levantamentos nacionais mostram baixos níveis de jovens ativos no Brasi1 ${ }^{6,9,10}$. Em se tratando da população escolar, a última pesquisa nacional de saúde do escolar demonstrou que 65,2\% dos adolescentes não atingiam a recomendação semanal de atividade física, e no estado do Paraná a inadequação foi de $61,5 \%{ }^{9}$.

Desta forma, o estímulo à prática de atividade física na adolescência é fundamental, podendo contribuir para que os adolescentes de maneira geral tenham uma vida mais ativa na vida adulta, fato esse conhecido como tracking da atividade física ${ }^{11}$. $\mathrm{O}$ tracking também pode ocorrer de uma maneira reversa, quando a inatividade física é iniciada na infância ou na adolescência, tornando-se mais difícil de modificar na vida adulta ${ }^{12}$. Dentre as variáveis que explicam o vínculo à prática de atividades físicas entre adolescentes, destaca-se o apoio social ${ }^{13}$. Esse apoio se caracteriza pela assistência oferecida, ou recursos postos à disposição por diferentes grupos de pessoas, tais como parentes, irmãos, pais e amigos ${ }^{14}$.

O apoio social de pais e amigos para a prática de atividade física é realizado de forma particular e distinta ${ }^{15,16}$. O apoio social cedido pelos pais, por exemplo, é voltado para a facilitação ao acesso de equipamentos esportivos, locais de prática esportiva e transporte ${ }^{15}$. O apoio social dos amigos, por sua vez, consiste na participação conjunta na prática de atividade física e incentivo à mesma ${ }^{16,17}$. Ambos desempenham influência social sobre a prática de atividade física dos adolescentes,tanto por meio de modelação do comportamento, quanto do fornecimento de apoio social ${ }^{18}$.

Considerando o tracking da atividade física, conhecer os efeitos do apoio social em adolescentes podem contribuir na elaboração de iniciativas para redução dos níveis da inatividade física nessa população. Poucos estudos epidemiológicos no Brasi ${ }^{18,19}$ envolvendo adolescentes escolares tentaram associar o apoio social de pais e amigos com o nível de atividade física, e apenas dois artigos no estado do Paraná avaliaram essa variável relacionada à atividade física. Porém a amostra foi composta apenas por escolares da rede pública de 14 a 18 anos do Ensino Médio, deixando de fora a faixa etária de 10 a 13 anos, que compreende as séries finais do Ensino Fundamental ${ }^{20-21}$. Diante disso, este estudo teve como objetivo verificar a associação entre nível de atividade física atual (lazer e deslocamento) e apoio social dos pais e amigos em escolares da rede pública.

\section{Métodos}

Essa pesquisa com delineamento transversal, foi aprovada pelo Comitê de Ética em Pesquisa da Universidade Estadual de Maringá, sob parecer número 1.453.730, em consonância com a Declaração de Helsinki. Esse estudo caracterizou-se como censo escolar, visto que envolveu toda população escolar do Ensino Fundamental II e Médio, composta por estudantes com idade variando entre 10 a 18 anos, das oito escolas públicas existentes no município de Paranavaí (PR). De acordo com dados de 2016 do Núcleo Regional de Educação deste município, um total de 3.483 escolares estavam matriculados nas oito escolas da cidade, e somente os escolares que apresentarem o Termo de Consentimento Livre e Esclarecido (TCLE) assinado pelos responsáveis foram avaliados $(\mathrm{n}=2.759)$.

A coleta de dados foi realizada nos meses de março e abril de 2016, durante o horário escolar por avaliadores previamente treinados.Para tanto, um estudo piloto foi conduzido em uma subamostra de 124 escolares com as mesmas características da amostra final, a qual foi realizada na semana que antecedeu a coleta de dados. Todos os erros das avaliações identificados foram imediatamente corrigidos e averiguados na primeira coleta de dados.

As variáveis analisadas no estudo foram: sociodemográficas (idade e sexo), nível de atividade física e apoio social dos pais e amigos. Todos os dados foram coletados por meio de questionário autoadministrado em sala de aula, o qual teve preenchimento supervisionado por um avaliador de modo contínuo e proximal, em cada turma. Todas as questões foram lidas e explicadas pelos avaliadores, e no caso de dúvidas por parte dos escolares, as perguntas foram sanadas em voz alta para que todos os demais pudessem ouvir.

O nível de atividade física foi mensurado por uma adaptação do questionário Self-Administered Physical Activity Checklist ${ }^{22,23}$. O ponto de corte adotado para inadequação do nível de atividade física foi de $<300 \mathrm{~min}$ por semana ${ }^{3}$. O apoio social foi avaliado por meio de uma escala de Apoio Social para Atividade Física em Adolescentes (ASAFA), a qual contém 10 itens $^{24}$, cinco deles são destinados a avaliar o apoio social dos pais e cinco ao apoio social dos amigos., considerando diferentes tipos de apoio (estimular, praticar, assistir, convidar, comentar, conversar e transportar ou fornecer transporte). Todos os itens estavam ancorados com escala Likert de quatro pontos (nunca $=1$; raramente $=2$; frequentemente $=3$; sempre $=4$ ), referentes à frequência com que os pais e os amigos ofereciam cada um dos tipos de apoio social durante uma semana típica. $\mathrm{O}$ ponto de corte para inadequação foi $<3$ pontos. 
A qualidade das informações obtidas pelo questionário foi feita por meio de aplicação replicada, com intervalo de uma semana entre elas, em uma subamostra.Os valores de reprodutibilidade foram calculados pelo coeficiente de correlação intraclasse (CCI) para as variáveis contínuas e o Índice Kappa para as dicotômicas. Nesse sentido para as variáveis contínuas os valores de $\mathrm{CCI}$ foram superiores a 0,8 , a saber: tempo de atividade física semanal $=0,83$; apoio social dos pais $=0,89$; apoio social dos amigos= 0,84 e apoio social dos pais e amigos $=0,81$. Para as variáveis dicotômicas os valores estiveram acima de 0,67 (tempo de atividade física semanal= 0,69; apoio social dos pais $=0,69$; apoio social dos amigos $=0,66 \mathrm{e}$ apoio social dos pais e amigos $=0,77$ ).

Para a análise estatística, foi usado o teste de Kolmogorov Smirnov para identificar a normalidade dos dados. A análise descritiva foi feita por medianas e intervalo interquartílico nas variáveis contínuas, e por frequência absoluta e relativa para as categóricas. $\mathrm{O}$ teste do Qui-quadrado foi adotado para verificar diferença na proporção de inatividade física (variável dependente), segundo as categorias das variáveis independentes(apoio social dos apaís e amigos). A regressão logística binária multivariável foi empregada para determinar a razão de chances (OR) e os respectivos intervalos de confiança (95\%), no intuito de analisar a associação de nível de atividade física (variável dependente) com as variáveis independentes. Todas as variáveis estudadas foram dicotomizadas e o critério para inclusão no modelo multivariado foi um nível de associação de $\mathrm{p} \leq 0,20$ com a variável dependente, pelo teste Qui-quadrado. As análises foram feitas por meio do Statistical Package for a Social Science (SPSS), versão 20.0, considerando-se $\mathrm{p}<0,05$.

\section{Resultados}

Essa pesquisa apresentou 20,8\% de perdas e recusas em relação ao total de escolares matriculados nas oito escolas da cidade $(n=3.483)$. Nesse sentido foram entrevistados 2.759 adolescentes (50,5\% meninas e 49,5\% meninos), os quais responderam todas as questões contidas nos questionários de atividade física e apoio social.

A tabela 2 compara os escolares insuficientes ativos e ativos em relação a idade e o tempo de atividade física semanal. Em relação a idade, apenas o sexo feminino apresentou diferença significativa $(\mathrm{p}=0,002)$, onde o grupo insuficiente ativo apresentou-se mais velho. Já no tempo de atividade física semanal, tanto no geral como em ambos os sexos, os resultados mostraram que o grupo ativo obteve tempo superior de atividade física em relação aos insuficientes ativos $(\mathrm{p}<0,001)$.

$\mathrm{Na}$ tabela 3, os resultados da analise de proporções verificou que a falta de apoio social oferecido por pais e amigos se associou de modo estatisticamente significante $(\mathrm{p} \leq 0,001)$ ao nível inadequado de atividade física (insuficientes ativos).

Os resultados da análise multivariável demonstraram que os escolares que referiram não receber apoio social adequado dos amigos e dos pais apresentaram 1,5 (IC95\%: 1,2-1,9) e 2,0 (IC95\%: 1,6-2,6) duas vezes mais, respectivamente, de serem insuficientemente ativos fisicamente em relação aos que tiveram apoio adequado, independente do sexo e idade.

Ao estratificar por sexo, a análise ajustada pela idade mostrou associação significativa no apoio geral apenas no sexo masculino, onde os escolares que não tiveram apoio social adequado tiveram 1,9 vezes mais chances (90\%) (IC95\%: 1,2-2,9) de serem insuficientemente ativos em relação aos escolares que tiveram apoio ade-

Tabela 1 - Idade e tempo de atividade física semanal em escolares insuficientes ativos e ativos de Paranavaí, 2016 ( $\mathrm{n}=2.759$ ).

\begin{tabular}{lcc}
\hline Variáveis & & Mediana (intervalo interquartílico) \\
\cline { 2 - 3 } & Insuficientemente ativos & Ativos \\
\hline Todos & $\mathrm{n}=1071$ & $\mathrm{n}=1688$ \\
Idade (anos) & $14,5(3,2)$ & $14,4(3,4)$ \\
TAF (minutos/semana) & $120(168)$ & $840(939)$ \\
Masculino & $\mathrm{n}=374$ & $\mathrm{n}=992$ \\
Idade (anos) & $14,3(3,4)$ & $14,5(3,3)$ \\
TAF (minutos/semana) & $120(170)$ & $930(1016)$ \\
Feminino & $\mathrm{n}=697$ & $\mathrm{n}=696$ \\
Idade (anos) & $14,5(3,1)$ & $14,2(3,4)$ \\
TAF (minutos/semana) & $108(165)$ & $690(750)$ \\
\hline
\end{tabular}

Insuficientemente ativos: Insuficientes ativos; TAF: Tempo de atividade física semanal. *Valores significativos: $\mathrm{p} \leq 0,05$. 
Tabela 2 - Apoio Social dos pais e amigos em relação ao nível de atividade física dos escolares de Paranavaí, 2016 ( $\mathrm{n}=2.759$ ).

\begin{tabular}{|c|c|c|c|c|c|c|}
\hline \multirow{2}{*}{ Variáveis } & & \multicolumn{2}{|c|}{ Todos } & \multicolumn{2}{|c|}{$\begin{array}{c}\text { Insuficientemente } \\
\text { ativos }\end{array}$} & \multirow[t]{2}{*}{$\mathrm{p}$-valor } \\
\hline & & $\mathrm{n}$ & $(\%)$ & $\mathrm{n}$ & (\%) & \\
\hline \multicolumn{7}{|c|}{ Todos } \\
\hline \multirow{2}{*}{ Apoio social dos pais } & Adequado & 761 & 27,6 & 168 & 22,1 & \multirow{2}{*}{$<0,001$} \\
\hline & Inadequado & 1999 & 72,4 & 904 & 45,2 & \\
\hline \multirow{2}{*}{ Apoio social dos amigos } & Adequado & 1048 & 38,0 & 267 & 25,5 & \multirow{2}{*}{$<0,001$} \\
\hline & Inadequado & 1711 & 62,0 & 804 & 47,0 & \\
\hline \multirow{2}{*}{ Apoio social dos pais e amigos } & Adequado & 938 & 34,0 & 203 & 21,6 & \multirow{2}{*}{$<0,001$} \\
\hline & Inadequado & 1822 & 66,0 & 869 & 47,7 & \\
\hline \multicolumn{7}{|c|}{ Masculino } \\
\hline \multirow{2}{*}{ Apoio social dos pais } & Adequado & 407 & 29,8 & 71 & 17,4 & \multirow{2}{*}{$<0,001$} \\
\hline & Inadequado & 959 & 70,2 & 304 & 31,7 & \\
\hline \multirow{2}{*}{ Apoio social dos amigos } & Adequado & 640 & 46,9 & 121 & 18,9 & \multirow{2}{*}{$<0,001$} \\
\hline & Inadequado & 726 & 53,1 & 253 & 35,0 & \\
\hline \multirow{2}{*}{ Apoio social pais e amigos } & Adequado & 559 & 41,0 & 90 & 16,1 & \multirow{2}{*}{$<0,001$} \\
\hline & Inadequado & 807 & 59,0 & 285 & 35,3 & \\
\hline \multicolumn{7}{|c|}{ Feminino } \\
\hline \multirow{2}{*}{ Apoio social dos pais } & Adequado & 353 & 25,3 & 97 & 27,5 & \multirow{2}{*}{$<0,001$} \\
\hline & Inadequado & 1040 & 74,7 & 600 & 57,7 & \\
\hline \multirow{2}{*}{ Apoio social dos amigos } & Adequado & 408 & 29,3 & 146 & 35,8 & \multirow{2}{*}{$<0,001$} \\
\hline & Inadequado & 985 & 70,7 & 551 & 55,9 & \\
\hline \multirow{2}{*}{ Apoio social pais e amigos } & Adequado & 378 & 27,1 & 113 & 29,9 & \multirow{2}{*}{$<0,001$} \\
\hline & Inadequado & 1015 & 72,9 & 584 & 57,5 & \\
\hline
\end{tabular}

Tabela 3 - Associação entre apoio social dos pais amigos e nível de atividade física em escolares de Paranavaí, medido por regressão logística (n=2.759).

\begin{tabular}{|c|c|c|}
\hline Variáveis & & Odds ratio (IC95\%)* \\
\hline \multicolumn{3}{|c|}{ Todos } \\
\hline \multirow{2}{*}{ Apoio social dos pais } & Adequado & 1 \\
\hline & Inadequado & $2,0(1,6-2,6)$ \\
\hline \multirow{2}{*}{ Apoio social dos amigos } & Adequado & 1 \\
\hline & Inadequado & $1,5(1,2-1,9)$ \\
\hline \multirow{2}{*}{ Apoio social pais e amigos } & Adequado & 1 \\
\hline & Inadequado & $1,6(1,2-2,1)$ \\
\hline \multicolumn{3}{|c|}{ Masculino } \\
\hline \multirow{2}{*}{ Apoio social dos pais } & Adequado & 1 \\
\hline & Inadequado & $1,4(1,0-2,1)$ \\
\hline \multirow{2}{*}{ Apoio social dos amigos } & Adequado & 1 \\
\hline & Inadequado & $1,4(1,0-2,0)$ \\
\hline \multirow{2}{*}{ Apoio social pais e amigos } & Adequado & 1 \\
\hline & Inadequado & $1,9(1,2-2,9)$ \\
\hline \multicolumn{3}{|c|}{ Feminino } \\
\hline \multirow{2}{*}{ Apoio social dos pais } & Adequado & 1 \\
\hline & Inadequado & $2,7(1,9-3,7)$ \\
\hline \multirow{2}{*}{ Apoio social dos amigos } & Adequado & 1 \\
\hline & Inadequado & $1,6(1,2-2,2)$ \\
\hline \multirow{2}{*}{ Apoio social pais e amigos } & Adequado & 1 \\
\hline & Inadequado & $1,4(1,0-1,4)$ \\
\hline
\end{tabular}

*Ajustado pela idade. 
quado. Para o apoio dos pais e amigos, apenas o sexo feminino teve associação, mostrando que meninas com ausência de apoio tiveram 2,7 (IC95\%: 1,9-3,7) e 1,6 (IC95\%: 1,2-2,2) mais chances de serem insuficientemente ativas respectivamente.

\section{Discussão}

Os principais achados do presente estudo mostram que,de maneira geral, os escolares de modo geral sem apoio social adequado de pais e/ou amigos tiveram maiores chances de serem insuficientemente ativos. Similarmente aos achados do presente estudo, em uma pesquisa realizada na cidade de Curitiba (PR) com 1.518 escolares entre 14 a 18 anos de idade, foi evidenciado que um maior fornecimento de apoio social da família e dos amigos esteve associado com o maior engajamento na prática de atividade física dos escolares em ambos os sexos ${ }^{20}$.

Neste sentido, nota-se que pais e amigos desempenham elevada influência social sobre a prática de atividade física dos adolescentes, tanto por meio de modelação do comportamento, quanto mediante o fornecimento de apoio social ${ }^{24}$. Assim, é necessário que o núcleo familiar e de amigos se envolvam no desenvolvimento da criança e do adolescente, apoiando o comportamento ativo, principalmente para que eles tenham confiança para adotar a atividade física em seu dia a dia. Com efeito, destaca-se que adolescentes com maior apoio social dos pais e dos amigos se sentem mais capazes de superar barreiras para a prática de atividades físicas ${ }^{25,26}$

O presente estudo observou que escolares do sexo masculino apresentaram maior apoio social de amigos do que dos pais $(46,9 \%$ tiveram apoio adequado de amigos e apenas 29,8\% exibiram apoio a que dado por parte dos pais). Do mesmo modo, um estudo com adolescentes escoceses mostrou que com o passar dos anos na adolescência o apoio social dos amigos aumenta ${ }^{27}$. Embora os motivos que explicam esse fenômeno não estejam bem estabelecidos, há indicativos de que a influência de amigos sobre a prática de atividade física pode ser dada por meio de diferentes apoios sociais, como o apoio em forma de práticas de atividades físicas coletivas e os comentários positivos sobre a mesma ${ }^{28}$. Adicionalmente, nessa faixa etária adolescentes passam várias horas do dia em contato diretocom os amigos, e desse modo pode ser que influencia exercida sobre a prática de atividade física possa ser maior em relação aos pais.

Esse estudo identificou ainda que escolares que reportaram apoio inadequado de seus pais e amigos apre- sentam cerca de $60 \%$ a mais de chances de serem insuficientemente ativos. Em relação ao apoio dos pais, escolares que não tiveram apoio adequado tiveram duas vezes mais chances de serem insuficientemente ativos quando comparados aos escolares que tiveram apoio adequado. Em relação ao apoio dos amigos, a magnitude da associação também foi elevada, independente do sexo e idade. Nesse contexto, há relevância elevada do apoio familiar e de amigos para a prática de atividades de adolescentes, independente do tipo de apoio. Pais e amigos, além de influenciarem diretamente na participação dos adolescentes na prática de atividade física, podem por meio do apoio social adequado fazer com que os adolescentes se sintam mais capazes de realizar atividades, ainda que haja presença de algumas barreiras ${ }^{18}$.

Ao estratificar por sexo, pode-se perceber que a chance das meninas serem insuficientemente ativas foi 2,7 (IC95\%: 1,9-3,7) vezes maior entre as que receberam apoio insuficiente dos pais e 1,6 (IC95\%: 1,2-2,2) vezes superior entre as que receberam apoio insuficiente dos amigos. Apesar dos intervalos de confiança se cruzarem, essa diferença na chance de atividade física insuficiente $(2,7$ vs. 1,6$)$ é relevante do ponto de vista epidemiológico e clínico, tendo em vista que estudos prévios envolvendo adolescentes brasileiros ${ }^{18-21}$ não encontraram valores discrepantes entre o apoio dos pais e amigos para o sexo feminino. De acordo com esse achado, duas perguntas se fazem necessárias: 1) Será que os pais não incentivam meninas da mesma forma que meninos para prática de atividade física? 2) Quais motivos levariam os pais em apoiar mais ou meninos em relação as meninas? Para as respostas dessas perguntas, pesquisas envolvendo os pais são necessárias.

Adicionalmente, essa pesquisa apresentou valores de reprodutibilidade satisfatórios nos dois questionários utilizados (atividade física e apoio social). Depois do estudo que validou em adolescentes brasileiros ${ }^{23}$, nenhuma outra pesquisa buscou replicar as avaliações, fato esse que impossibilita inferências acerca dos valores de confiabilidade das medidas. No entanto, os valores de CCI desse estudo $(0,83)$ para tempo de atividade física se assemelharam ao estudo de validação $(0,88)$. Da mesma forma, para o apoio social os valores de CCI variaram entre 0,81 e 0,89 , valores esses próximos a pesquisa de validação $(0,89$ a 0,91$)$.

Os resultados apresentados nessa pesquisa implicam em importantes aplicações práticas, como a necessidade de novos estudos, especificamente na faixa etária que envolve o período da adolescência (10 a 18 anos de idade), no intuito de melhor compreender as possíveis 
associações entre apoio social e nível de atividade física. Apesar do alto custo e do tempo necessário para a coleta de dados, existe ainda necessidade de estudos epidemiológicos que avaliem o nível de atividade física de maneira direta, por exemplo, o uso de acelerômetros. Novos estudos com variáveis de ajuste (IMC, circunferência de cintura, nível socioeconômico, etnia, maturação e tempo de tela) são necessários para poder explicar quais são as variáveis que podem interferir, principalmente na prática de atividade física. Por fim, políticas públicas para o incentivo a prática de atividade física devem encorajar o fornecimento de diferentes tipos de apoio social não só dos pais e dos amigos, mas em diferentes contextos como: postos de saúde, escolas, igrejas, e locais públicos.

O presente estudo tem algumas limitações, dentre as quais destacam-se: a medida do nível de atividade física foi realizada por meio de questionário, o qual pode superestimar ou subestimar o real tempo de prática, apesar do instrumento ser validado para amostra envolvida nesta investigação; trata-se de um estudo transversal, a qual não permite estabelecer relação causal entre apoio social e nível de atividade física. Entretanto, este estudo também apresenta alguns pontos fortes: foi realizado um censo da população alvo do estudo, tendo em vista que todas as escolas e todas as turmas foram avaliadas; houve uma baixa taxa de perdas e recusas, o que pode contribuir para maior fidedignidade aos achados aqui encontrados. Por fim, essa pesquisa pioneiramente levanta algumas indagações frente ao apoio social dos pais ser diferente em meninas e meninos, indicando assim a necessidade de pesquisas que avaliem potencias motivos para essa diferença.

Conclui-se que, entre adolescentes escolares da rede pública de ensino de Paranavaí, existe associação entre nível de atividade física e apoio social de pais e amigos. Escolares que apresentaram apoio social inadequado, tanto dos pais como de amigos, tiveram maiores chances de serem insuficientemente ativos. Esses achados indicam que a promoção da prática de atividade física em adolescentes deve incluir ações que favoreçam o fornecimento de apoio social dos pais e dos amigos, incentivando os escolares de diferentes formas, para que eles adotem a prática de atividade física em seu estilo de vida.

\section{Conflito de interesses}

Os autores declaram não haver conflito de interesses.

\section{Financiamento}

Fundação Auraucária de apoio ao Desenvolvimento Científico e Tecnológico do Estado do Paraná.

\section{Contribuição dos autores}

Todisco WMD, concebeu o projeto do artigo, participou da coleta de dados, tabulações e análises dos dados e da redação do manuscrito. Guilherme FR, concebeu o projeto do artigo, auxiliou na análises dos dados, produção textual e revisão crítica do trabalho. Molena -Fernandes CA, auxiliou na produção textual, Santos SR, participou da coleta de dados, tabulações e redação do manuscrito. Ravagnani RJF, participou da coleta de dados, tabulações e redação e revisão do manuscrito. Barbosa WA, participou da coleta de dados e revisão crítica do trabalho. Del Vecchio FB, auxiliou no desenvolvimento do projeto, participação na produção textual e revisão crítica. Rinaldi $\mathrm{W}$, auxiliou no desenvolvimento do projeto, produção textual e revisão crítica.

\section{Agradecimentos}

A equipe de aprimoramento e incentivo a produção científica em nível Superior (Capes), Brasil, ao Núcleo Regional de Educação - Paranavaí e todos as Instituições Públicas de Ensino participantes da pesquisa.

\section{Referências}

1. Caspersen CJ, Powell KE, Christenson GM. Physical activity, exercise, and physical fitness: definitions and distinctions for health-related research. Public Health Rep. 1985;100(2):126-31.

2. Strong WB, Malina RM, Blimkie CJR, Daniels SR, Dishman RK, Gutin B, et al. Evidence Based Physical Activity for School-age Youth. J Pediatr. 2005;146(6):723-27.

3. Florindo AA, Hallal PC, Moura EC, Malta DC. Prática de atividades físicas e fatores associados em adultos, Brasil, 2006. Rev Saude Publica. 2009;43(Supl 2):65-73.

4. Janssen I, Leblanc A. Systematic Review of the Health Benefits of Physical Activity and Fitness in School-Aged Children and Youth. Int J Behav Nutr Phys Act. 2010;7 (40):183-219.

5. Hallal PC, Knuth AG, Cruz DKA, Mendes MI, Malta DC. Prática de atividade física em adolescentes brasileiros. Cien Saude Colet. 2010;15(2):3035-42.

6. Lee IM, Shiroma EJ, Lobelo F, Puska P,Blair SN, Katzmarzyk PT. Effect of physical inactivity on major non-communicable diseases worldwide: an analysis of burden of disease and life expectancy. Lancet. 2012;380(9838):219-29.

7. Ciolac EG, Guimarães GV. Exercício físico e síndrome metabólica. Rev Bras Med do Esporte. 2004;10(4):319-30.

8. Sasaki RSA, Leles CR, Malta DC, Sardinha LMV, Freire CM. Prevalência de relação sexual e fatores associados em adolescentes escolares de Goiânia, Goiás, Brasil. Cien Saude Colet. 2015;20(1):95-104.

9. Ministério da Saúde, IBGE. Pesquisa Nacional de Saúde do Escolar 2015. Instituto Brasileiro de Geografa e Estatística. 2016. 132 p. Disponível em: https://biblioteca.ibge.gov.br/ visualizacao/livros/liv97870.pdf.

10. Tassitano RM, Bezerra J, Tenório MCM, Colares V, Barros MVG, Hallal PC. Physical activity in brazilian adolescents: A systematic review. Rev Bras Cineantropometria e Desempenho Hum. 2007;9(1):55-60.

11. Hallal PC, Andersen LB, Bull FC, Guthold R, Haskell W, Ekelund U. - Global physical activity levels: surveillance progress, pitfalls, and prospects. Lancet. 2012 380(9838):247-57. 
12. Maia JAR, Lefevre J, Claessens A, Renson R, Vanreusel B, Beunen G. Tracking of physical fitness during adolescence: a panel study in boys. Med Sci Sports Exerc. 2001;3395):765-71.

13. Motl RW, Dishman RK, Saunders RP, Dowda M, Pate RR. Perceptions of physical and social environment variables and self-efficacy as correlates of self-reported physical activity among adolescent girls. J Pediatr Psychol. 2007;32(1):6-12.

14. Horst KV, Paw MJCA, Twisk JWR, Mechelen WV. A Brief Review on Correlates of Physical Activity and Sedentariness in Youth. Med Sci Sport Exerc. 2007;39(8):1241-50.

15. Duncan SC, Duncan TE, Strycker LA. Sources and types of social support in youth physical activity. Health Psychol. 2005;24(1):3-10.

16. Morrissey JL, Wenthe PJ, Letuchy EM, Levy SM, Janz KF. Specific types of family support and adolescent non-school physical activity levels. Pediatr Exerc Sci. 2012;24(3):333-46.

17. Sierra H, Cordova M, Chen C-SJ, Rajadhyaksha M. Confocal Imaging-Guided Laser Ablation of Basal Cell Carcinomas: An Ex Vivo Study. J Invest Dermatol. 2015;135(2):612-15.

18. Cheng LA, Mendonça G, Farias Júnior JC De. Physical activity in adolescents: analysis of the social influence of parents and friends. J Pediatr (Rio J). 2013;90(1):35-41.

19. Ricardo L, Rombaldi A, Otte J, Perez A, Azevedo M. Association between social support and leisure-time physical activity among high school students. Rev Bras Atividade Física Saúde. 2013;18(2):168-76.

20. Prado CV, Lima AV, Fermino RC, Añez CRR, Reis RS. Apoio social e prática de atividade física em adolescentes da rede pública de ensino: qual a importância da família e dos amigos? Cad Saude Publica. 2014;30(4):827-38.

21. Fermino RC, Rech CR, Hino AAF, Rodriguez Añez CR, Reis RS. Atividade física e fatores associados em adolescentes do ensino médio de Curitiba, Brasil. Rev Saude Publica. 2010;44(6):986-95.
22. Sallis JF, Strikmiller PK, Harsha DW, Feldaman HA, Ehlinger S, Stone EJ, et al. Validation of interviewer- and self- administered physical activity checklists for fifth grade students. Med Sci Sports Exerc. 1996;28(7):840-51.

23. Farias Júnior JC, Lopes A da S, Mota J, Santos MP, Ribeiro JC, Hallal PC. Validade e reprodutibilidade de um questionário para medida de atividade física em adolescentes: uma adaptação do Self-Administered Physical Activity Checklist. Rev Bras Epidemiol.2012;15(1):198-210.

24. Lima AV, Fermino RC, Oliveira MP, Rodriguez Añez CR, Reis RS. Distância percebida até as instalações de lazer e sua associação com a prática de atividade física e de exercícios em adolescentes de Curitiba, Paraná, Brasil. Cad Saude Publica. 2013;29(8):1507-21.

25. Silva JVP. (In)Atividade física na adolescência: revisão sistemática. / Physical (in) activity in adolescence: a systematic review. Rev Bras Ciênc e Mov. 2013;21(3):166-79.

26. Dishman RK, Saunders RP, Motl RW, Dowda M, Pate RR. Self-efficacy moderates the relation between declines in physical activity and perceived social support in high school girls. J Pediatr Psychol. 2009;34(4):441-51.

27. Kirby J, Levin K, Inchley J. Parental and peer influences on physical activity among Scottish adolescents: a longitudinal study. J Phys Act Heal. 2011;8(6):785-93.

28. Mendonça G, Farias Júnior JC. Physical activity and social support in adolescents: analysis of different types and sources of social support. J Sports Sci. 2015;33(18):1942-51.

Recebido: 20/07/2017

Aprovado: 11/12/2017

\section{Como citar este artigo:}

Todisco WMD, Guilherme FR, Molena-Fernandes CA, Ravagnani RJF, Barbosa WA, Del Vecchio FB, Rinaldi W. Nivel de atividade física e apoio social dos pais e amigos em escolares da rede pública. Rev Bras Ati Fis Saúde. 2017; 22(5):457-63. DOI: 10.12820/rbafs. v.22n5p457-63 